\title{
Brillouin scattering-like effect and non- reciprocal propagation of elastic waves due to spatio-temporal modulation of electrical boundary conditions in piezoelectric media
}

\begin{tabular}{|c|c|}
\hline Item Type & Article \\
\hline Authors & $\begin{array}{l}\text { Croënne, C.; Vasseur, J. O.; Bou Matar, O.; Ponge, M.-F.; Deymier, } \\
\text { P. A.; Hladky-Hennion, A.-C.; Dubus, B. }\end{array}$ \\
\hline Citation & $\begin{array}{l}\text { Brillouin scattering-like effect and non-reciprocal propagation } \\
\text { of elastic waves due to spatio-temporal modulation of electrical } \\
\text { boundary conditions in piezoelectric media 2017, } 110 \text { (6):061901 } \\
\text { Applied Physics Letters }\end{array}$ \\
\hline DOI & $10.1063 / 1.4975680$ \\
\hline Publisher & AMER INST PHYSICS \\
\hline Journal & Applied Physics Letters \\
\hline Rights & Published by AIP Publishing. \\
\hline Download date & 27/07/2021 06:47:12 \\
\hline Version & Final published version \\
\hline Link to Item & http://hdl.handle.net/10150/623049 \\
\hline
\end{tabular}




\section{Brillouin scattering-like effect and non-reciprocal propagation of elastic waves due to spatio-temporal modulation of electrical boundary conditions in piezoelectric media}

C. Croënne, J. O. Vasseur, O. Bou Matar, M.-F. Ponge, P. A. Deymier, A.-C. Hladky-Hennion, and B. Dubus

Citation: Appl. Phys. Lett. 110, 061901 (2017); doi: 10.1063/1.4975680

View online: http://dx.doi.org/10.1063/1.4975680

View Table of Contents: http://aip.scitation.org/toc/apl/110/6

Published by the American Institute of Physics

\section{Articles you may be interested in}

High harmonic generation in $\mathrm{ZnO}$ with a high-power mid-IR OPA

Appl. Phys. Lett. 110, 061101061101 (2017); 10.1063/1.4975362

Low-frequency ultrasonic Bessel-like collimated beam generation from radial modes of piezoelectric transducers Appl. Phys. Lett. 110, 064101064101 (2017); 10.1063/1.4975800

Magnetic field sensor based on coupled photonic crystal nanobeam cavities

Appl. Phys. Lett. 110, 061110061110 (2017); 10.1063/1.4975804

Controlling the optical spin Hall effect with light

Appl. Phys. Lett. 110, 061108061108 (2017); 10.1063/1.4975681

Ferroelectric, pyroelectric, and piezoelectric properties of a photovoltaic perovskite oxide Appl. Phys. Lett. 110, 063903063903 (2017); 10.1063/1.4974735

Characterizing tips suitable for atomic force microscopy and spectroscopy with atomic resolution and spin sensitivity

Appl. Phys. Lett. 110, 061601061601 (2017); 10.1063/1.4973740
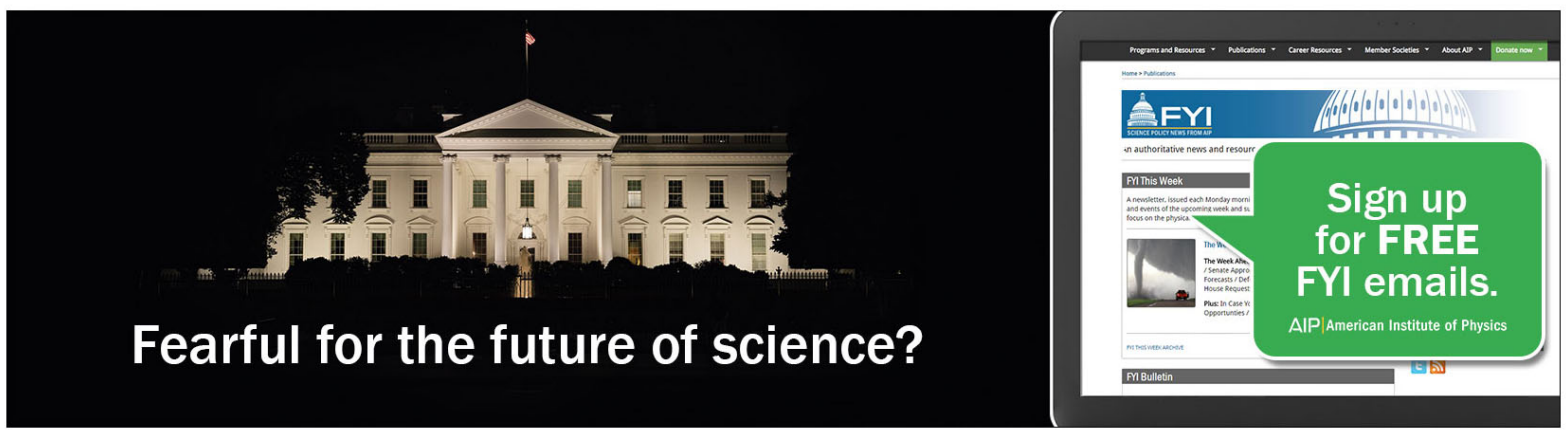


\title{
Brillouin scattering-like effect and non-reciprocal propagation of elastic waves due to spatio-temporal modulation of electrical boundary conditions in piezoelectric media
}

\author{
C. Croënne, ${ }^{1}$ J. O. Vasseur, ${ }^{1}$ O. Bou Matar, ${ }^{1}$ M.-F. Ponge, ${ }^{2}$ P. A. Deymier, ${ }^{3}$ \\ A.-C. Hladky-Hennion, ${ }^{1}$ and B. Dubus ${ }^{1}$ \\ ${ }^{1}$ CNRS, Centrale Lille, ISEN, Univ. Lille, Univ. Valenciennes, UMR 8520 - IEMN, F-59000 Lille, France \\ ${ }^{2}$ Département APY (UMR CNRS 5295), Institut de Mécanique et d'Ingénierie, Université Bordeaux I, 351 \\ cours de la Libération, 33405 Talence Cedex, France \\ ${ }^{3}$ Department of Materials Science and Engineering, University of Arizona, Tucson, Arizona 85721, USA
}

(Received 24 October 2016; accepted 20 January 2017; published online 7 February 2017)

\begin{abstract}
The properties of a one-dimensional phononic crystal made of identical piezoelectric elements separated by thin metallic electrodes connected to the ground are studied theoretically for cases where the locations of the electrical connections change as a function of time with a specific speed. This spatiotemporal modulation of the electrical boundary conditions results in significant non-linear effects that are evidenced numerically. The interaction between an incident harmonic longitudinal wave and the time-dependent phononic crystal is shown to lead to frequency splitting analogous to Brillouin scattering. Moreover, the boundaries of the Bragg bandgaps are strongly affected, and for some specific modulation speed, one-way wave propagation can be achieved. Published by AIP Publishing.

[http://dx.doi.org/10.1063/1.4975680]
\end{abstract}

Since the seminal work of Lord Rayleigh in $1887,{ }^{1}$ the propagation of waves in media exhibiting a periodic modulation of their physical properties has received a great deal of attention. When the modulation occurs in the spatial domain, these media correspond to periodic distributions of scatterers embedded in a host matrix and are nowadays known as photonic crystals ${ }^{2}(\mathrm{PtCs})$ for electromagnetic waves and phononic crystals $^{3}(\mathrm{PnCs})$ for acoustic waves. Due to the spatially periodic structure, their transmission spectra may present bandgaps in the frequency domain whereby propagation is forbidden. However, the periodic modulation may also occur in the temporal domain. For example, it was shown ${ }^{4}$ that a bulk medium whose dielectric function obeys a sinusoidal function of time with frequency $\mathcal{F}$ exhibits a photonic band structure periodic in the frequency domain with periodicity $\mathcal{F}$ and bandgaps in the wavenumber domain. Moreover, when a slab of this medium is illuminated at frequency $f_{0}$ at normal incidence, the reflected and transmitted waves contain harmonics of $\mathcal{F}$, and the slab itself behaves as a polychromatic source of frequencies $f_{n}^{ \pm}= \pm f_{0}+n \mathcal{F}, n$ being an integer. Similar results were obtained when considering the propagation of acoustic waves through a corrugated cylindrical waveguide with a time-varying diameter ${ }^{5}$ or through a fluid with periodically time-varying density and bulk modulus. ${ }^{6}$ One may note that the interaction between an incident wave and a time-varying propagating medium leads to a frequency splitting that resembles Brillouin scattering ${ }^{7,8}$ where the frequency shift between the scattered photon, $f^{\prime}$, and incoming photon, $f_{0}$, depends on the pump phonon frequency $\mathcal{F}$. This frequency shift is independent of the speed of wave in the propagating medium in contrast with Doppler-type effects observed in time-dependent PtCs and PnCs. ${ }^{9,10}$ The modulation of the physical characteristics of the propagating medium may also depend on both time and spatial coordinates. The effect of such spatio-temporal modulations on wave propagation was investigated in the context of parametric amplification ${ }^{11}$ in a linear transmission line made of a series of LC resonators with distributed time-space periodic shunt capacitances. More recently, the spatio-temporal modulation of the dielectric ${ }^{12,13}$ or acoustic ${ }^{14-16}$ properties of photonic or phononic structures has also been used to achieve time reversal symmetry breaking and then one-way wave propagation.

The present work focuses on the propagation of longitudinal acoustic waves in PnCs constituted of a periodic array of electrodes arranged in a homogeneous piezoelectric material. It is known that for such a system, ${ }^{17}$ if the electrodes are connected to the ground, a superlattice is formed which may exhibit frequency domain bandgaps at the edge of the Brillouin zone, whereas if all electrodes are left floating they have negligible effect on the propagation properties, provided that they are thin enough with respect to the wavelength. Here, we address the case of time-dependent electrical boundary conditions applied to the electrodes. We show theoretically with the help of the Finite Difference Time Domain (FDTD) method $^{18}$ that a spatio-temporal modulation of the piezoelectric PnC leads to a Brillouin scattering-like frequency splitting. Additionally, the system becomes strongly non-reciprocal, and for specific modulation characteristics, one-way wave propagation can be achieved.

The structure under study is a one dimensional stack of identical Lead Zirconate Titanate (PZT) ${ }^{19}$ elements separated by thin electrodes. Among this periodic set of electrodes, a periodic subset is connected to the ground and this subset is spatially shifted as a function of time. We can define a spatial period $a$ for this subset, as well as a constant shift velocity $v$, with a sign depending on the shift direction $(v>0$ for shifts towards $+x$, as defined in Fig. 1(a)). It should be pointed out that we do not consider electrodes moving at velocity $v$, but rather motionless electrodes connected to the ground through switches whose on/off states 
(a)
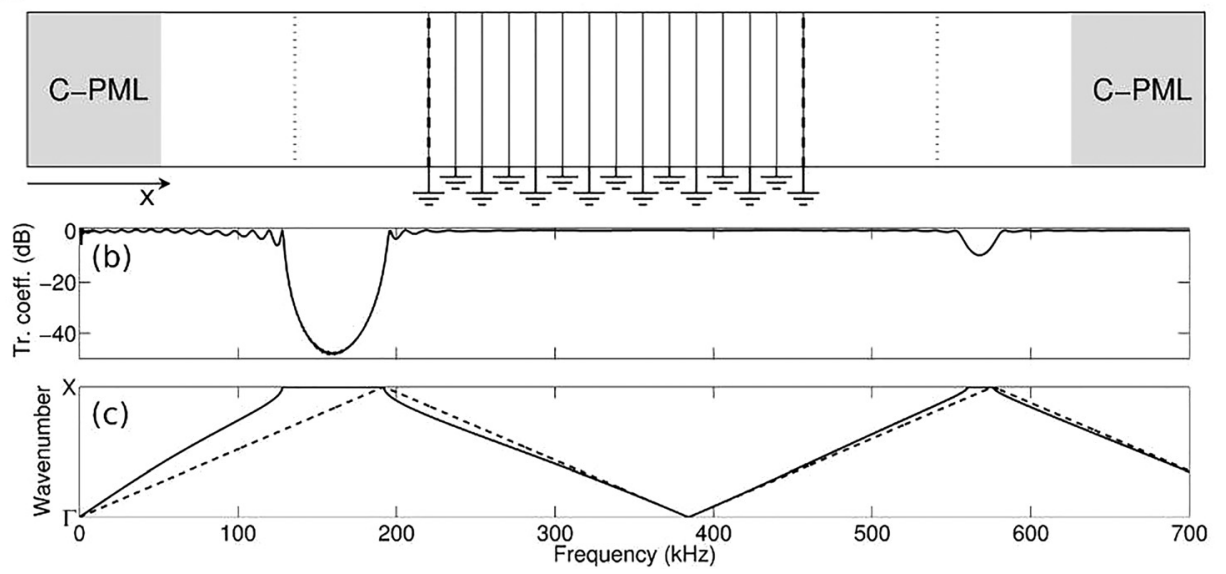

FIG. 1. (a) Piezoelectric stack geometry. 14 electrodes (thin lines) with $a=1 \mathrm{~cm}$ separation are grounded in the crystal region (delimited by two dashed lines). On each side, a measurement point $(\mathrm{A}$ or $\mathrm{B})$ is placed, $5 \mathrm{~cm}$ away from the crystal region boundary and from the C-PML boundary. CPMLs are $5 \mathrm{~cm}$-wide. (b) Transmission coefficient from A to B. (c) Band structure of an infinite piezoelectric $\mathrm{PnC}$ with electrodes located every $1 \mathrm{~cm}$ and connected to the ground (solid lines) or left floating (dashed lines). shift as a function of time with velocity $v$. In the following, to facilitate the interpretations, it is assumed that an electrode is present at each spatial location in the FDTD mesh, allowing for a smooth spatial shift of the grounded electrode conditions.

In the frame of electrostatics and linear elasticity, propagation of elastic waves is governed by Newton's law and equations of piezoelectricity. ${ }^{17}$ In the course of the FDTD calculations, these equations are discretized with the Yee algorithm which involves half-period shifted space and time grids for stress and elastic displacement. ${ }^{18}$ Specific ConvolutionPerfectly Matched Layers ${ }^{20}$ (C-PMLs) are used at both ends of the discretization mesh to alleviate unwanted reflection of waves on these ends (see Fig. 1(a)). The spatial mesh size $\Delta x=250 \mu \mathrm{m}$ is kept constant in all simulations, whereas the temporal mesh is chosen to both satisfy the CourantFriedrichs-Lewy criterion ${ }^{18}$ and give an integer number of time steps between two switching times. Figure 1(b) presents the normalized FDTD transmission coefficient through a stack made of 14 periods of the time-independent $1 \mathrm{D}$ piezoelectric $\mathrm{PnC}$, i.e., when the electrical connections never switch $(v=0)$, with $a=1 \mathrm{~cm}$. For the transmission calculation, two reference locations A and B are defined (see Fig. 1(a)). A is the mechanical stress source location, while B is located on the other side of the crystal. The first and last grounded electrode locations define the extent of the crystal region. The band structure of the same PnC but of infinite extent along the $x$ direction $^{17}$ is shown in Fig. 1(c). It exhibits a large bandgap from 129 to $192 \mathrm{kHz}$ approximately (width equal to $40 \%$ of the central frequency) and a narrow bandgap around $570 \mathrm{kHz}$. As expected, bandgaps observed in Fig. 1(c) correspond to the regions of very low transmission in Fig. 1(b). From Fig. 1(c), one notes that when all the electrodes are left floating, no bandgap occurs in the band structure and the PnC behaves as a non dispersive medium.

When $v \neq 0$, it is necessary to ensure that the whole set of grounded electrodes stays in the same region of the system and thus never reaches $\mathrm{A}$ or $\mathrm{B}$. Therefore, anytime a grounded electrode position reaches a boundary of the crystal (shown with dashed lines in Fig. 1(a)), and it is transferred to the opposite boundary. Figure 2(a) shows the temporal Fourier transform (FT) magnitude of the stress field at location B measured over a total simulation time of $500 \mu \mathrm{s}$ for three values of $v$, with an harmonic excitation of frequency $f_{0}=100 \mathrm{kHz}$ at location $\mathrm{A}$. For $v=0$, the system is linear and the FT shows a single peak (whose width is directly related to the total simulation time) at $f_{0}$. For $v \neq 0$, additional peaks appear at frequencies $f_{n}^{ \pm}= \pm f_{0}+n f_{v}, n$ being an integer and $f_{v}=v / a$ the characteristic frequency of the electrode connection changes, as $1 / f_{v}$ is the time interval between two equivalent states of the electrode connection schemes. This non-linear effect can be regarded as an

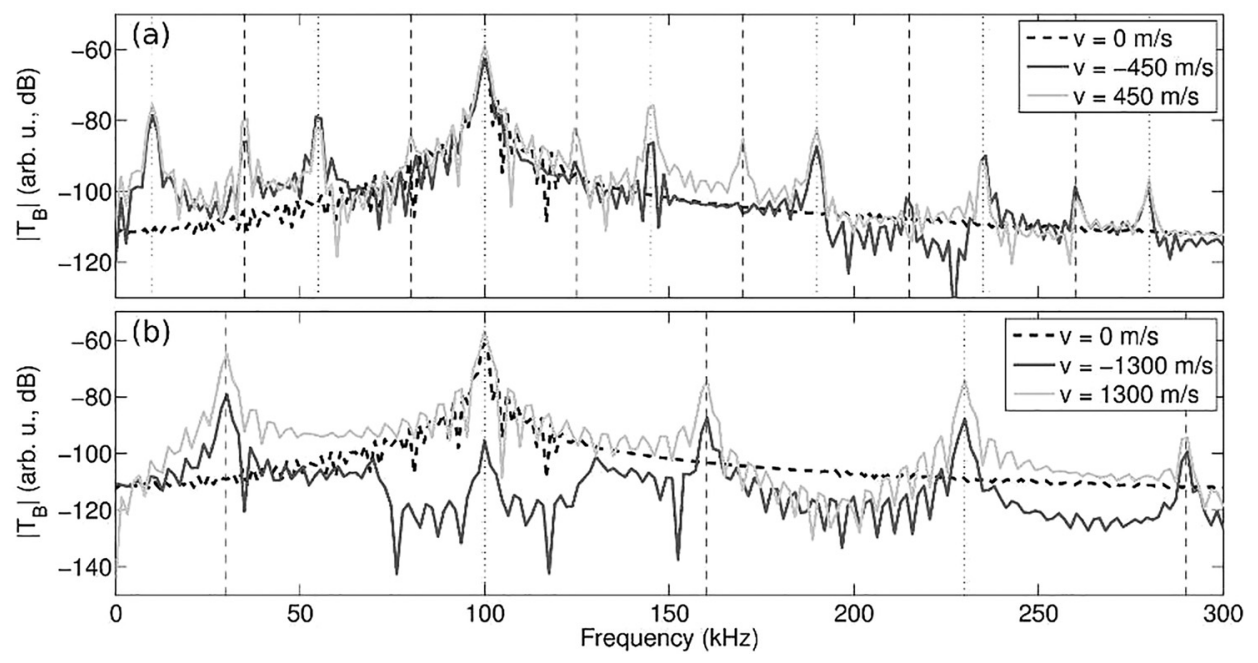

FIG. 2. Temporal FT of the stress field at location B, $T_{B}$ for (a) $v= \pm 450 \mathrm{~m} / \mathrm{s}$ and (b) $v= \pm 1300 \mathrm{~m} / \mathrm{s}$. Result for the time-independent crystal (i.e., for $v=0$ ) is shown on each graph for reference. The vertical dashed and dotted lines correspond to frequencies $f_{n}^{+}=$ $f_{0}+n f_{v}$ and $f_{n}^{-}=-f_{0}+n f_{v}$, respectively, with $f_{0}$ the excitation frequency, $f_{v}$ the characteristic frequency of the electrode connection changes, and $n$ an integer. 


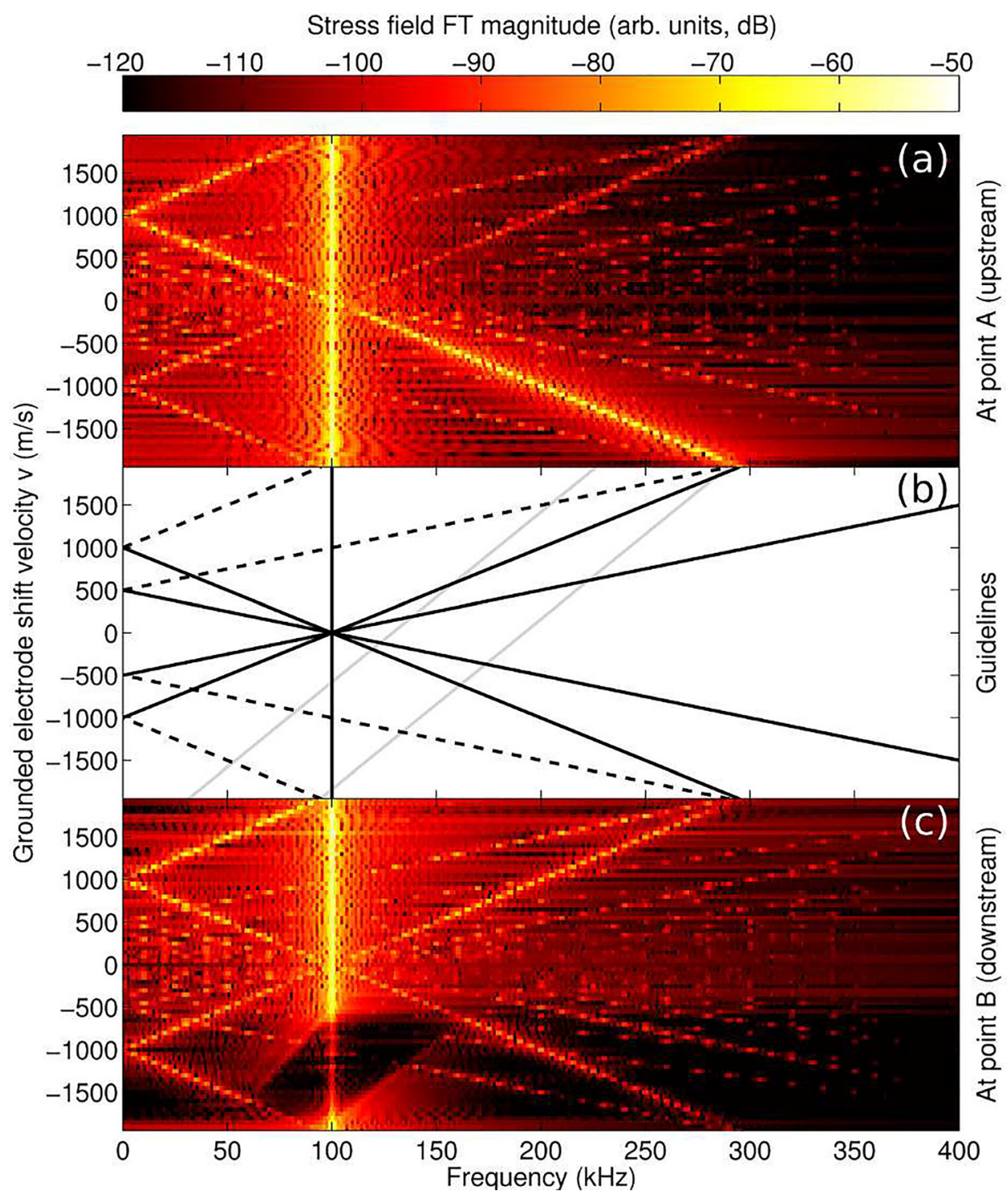

FIG. 3. Temporal FT of the stress field at locations (a) A and (c) B as a function of $v$, the grounded electrode shift velocity, for an excitation frequency $f_{0}=100 \mathrm{kHz}$. (b) Guidelines showing the interpretation of the main features in graphs (a) and (c). The solid and dashed black lines correspond to the Brillouin scattering-related peaks $f_{n}^{+}$ and $f_{n}^{-}$, respectively (with the same notations as in Figure 2). The solid gray lines show the expected modification of the first Bragg gap frequency boundaries considering the approximate dispersion diagram for the timedependent crystal (see the supplementary material). intermodulation between an acoustic probe of frequency $f_{0}$ and a pump of frequency $f_{v}$. It is also analogous to Brillouin scattering, ${ }^{7}$ if we regard the continuous shift of the electrical connections as a propagating local modulation of the effective material properties. The incident acoustic signal and the continuous shift are then analogous to Brillouin's probe photon and pump phonon, respectively, and the $f_{n}^{ \pm}= \pm f_{0}+n f_{v}$ formula simply corresponds to an energy conservation condition for their interaction. Figure 3 shows the same FT results, this time both at locations $\mathrm{A}$ and $\mathrm{B}$, and for the values of $v$ varying between -2000 and $+2000 \mathrm{~m} / \mathrm{s}$. Each horizontal line corresponds to a stress field FT obtained from an individual FDTD simulation. Figure 3(b) shows the lines corresponding to the $f_{n}^{ \pm}= \pm f_{0}+n f_{v}$ formula for $n$ varying between -2 and +2 . It can be verified that they match with the FT peaks particularly well, even for large values of $v$. Lines of lower amplitude are also visible, corresponding to larger values of $n$.

Classical Brillouin scattering effects require both an energy conservation and a momentum conservation relationship. To investigate the latter, we report in Figure 4 the spatial FT of the stress field inside the crystal region, for $v=1300 \mathrm{~m} /$ $\mathrm{s}$. Since the field data come from the FDTD simulations, it is in fact a time-space FT over the crystal region and over the full simulation time. The brightest spot in this figure corresponds to the incoming signal at $f_{0}$. The wavenumber for this spot $k^{\prime}\left(f_{0}\right)$ is close to the value obtained from the dispersion curve of the time-independent crystal $\left(k\left(f_{0}\right)\right.$, approximately $0.74 \pi / a)$. Several secondary bright spots also appear in the FT results, all at frequencies $f_{n}^{ \pm}= \pm f_{0}+n f_{v}$. They can be divided into two families. One family (highlighted with circled markers and connected with dark gray solid lines in Fig. $4)$ is formed by the two sets $\left(k_{n}^{+}, f_{n}^{+}\right)$and $\left(k_{n}^{-}, f_{n}^{-}\right)$with $k_{n}^{ \pm}= \pm k^{\prime}\left(f_{0}\right)+n G$, where $G$ is the reciprocal lattice vector norm $2 \pi / a$ and $n$ is an integer. This formula corresponds to the momentum conservation relationship. Indeed, since the grounded electrode subset possesses a spatial periodicity $a$, it can only exchange momentum in quanta of $G$. Therefore, this family of spots is a signature of the Brillouin scatteringrelated non-linear effect in the time-dependent crystal. The second family of spots lies mainly on two almost straight lines originating from $(k, f)=(0,0)$. Using the properties of eigenmodes in time-space periodic structures and some approximate dispersion formulas deduced from the simulated spatial FTs, it is possible to build an approximation of the dispersion curves for the time-dependent $\mathrm{PnC}$ (see 


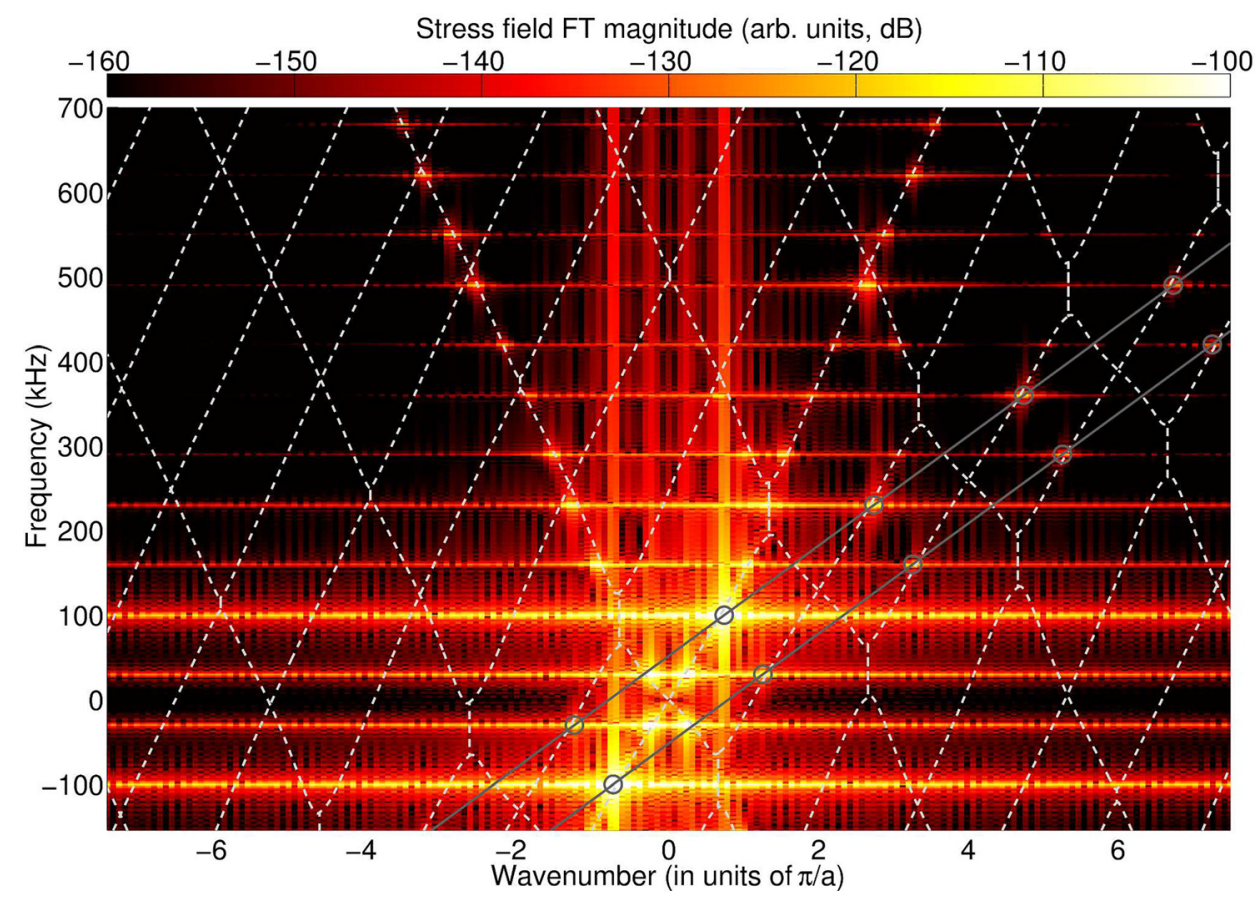

FIG. 4. Time-space FT of the stress field inside the crystal region for $v=$ $+1300 \mathrm{~m} / \mathrm{s}$ and $f_{0}=100 \mathrm{kHz}$. Light gray dashed lines show the approximate band diagram for the timedependent crystal, whereas dark gray solid lines with circle markers highlight the Brillouin scattering-related bright spots $\left(k_{n}^{ \pm}, f_{n}^{ \pm}\right)$. supplementary material). They are shown as light gray dashed lines in Fig. 4. It can be verified that the bright spots of both families are very close to the approximate curves. One possible explanation for the presence of two families of bright spots is that both Brillouin scattering-related and samefrequency phonon interactions are involved here. In practice, the Brillouin scattering-related process would transfer energy to other frequencies, whereas the same-frequency interaction would only change the wavenumber.

Reciprocity of fundamental frequency transmission coefficient (FFTC) is now investigated. Since the geometry is symmetric with respect to its center, comparing FFTCs at $f_{0}$ from $\mathrm{A}$ to $\mathrm{B}$ with two opposite values of $v$ is equivalent to comparing FFTCs from $\mathrm{A}$ to $\mathrm{B}$ and $\mathrm{B}$ to $\mathrm{A}$ at $f_{0}$ with a single value of $v$. Therefore, the results shown in Fig. 2(b), where the signal at $f_{0}$ for $v=-1300 \mathrm{~m} / \mathrm{s}$ is about $35 \mathrm{~dB}$ below that for $v=+1300 \mathrm{~m} / \mathrm{s}$, show that a highly non-reciprocal FFTC is achieved. The same effect is also clearly visible in Fig. 3 at location $\mathrm{B}$, with a signal at $f_{0}$ significantly reduced when $v$ is between -1900 and $-600 \mathrm{~m} / \mathrm{s}$ approximately, compared to the signal between +600 and $+1900 \mathrm{~m} / \mathrm{s}$.

We now focus on the dependence of the FFTC upon this choice of $f_{0}$. Figure 5 displays simulated FFTCs, for a $f_{0}$ parameter sweep between 10 and $700 \mathrm{kHz}$ with $10 \mathrm{kHz}$ steps, and a $v$ parameter sweep between -2000 and $+2000 \mathrm{~m} / \mathrm{s}$ with $50 \mathrm{~m} / \mathrm{s}$ steps. Each pixel of the image corresponds to an individual FDTD simulation. This parametric study shows that the previously observed FFTC dip results from a shift of the first Bragg bandgap (located at the $X$ point in the band diagram, i.e., at $k=\pi / a$ ) towards lower or higher frequencies depending on $v$. Even though its width and depth change slowly as a function of $v$, the boundaries of this apparent Bragg bandgap are close to the ones deduced from the approximate dispersion diagram for the time-dependent crystal (see supplementary material), shown in Fig. 5 as two oblique light gray lines. Interestingly, the effect is not limited to

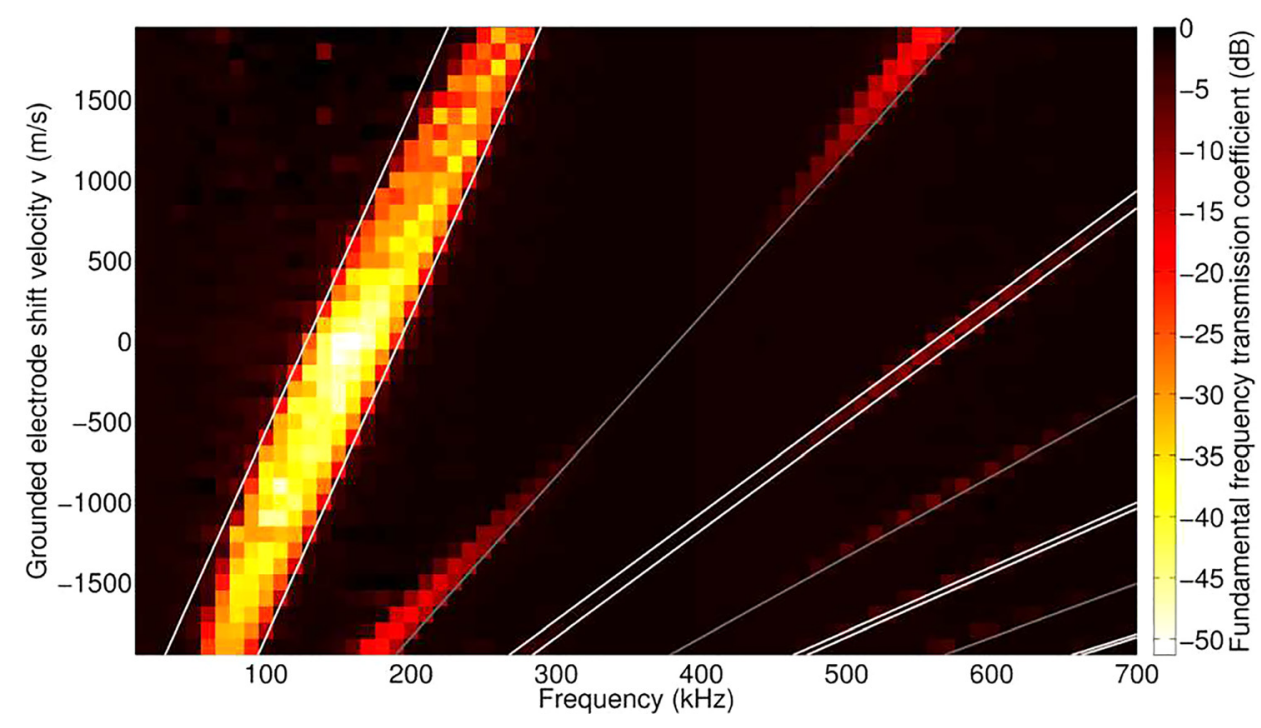

FIG. 5. FFTC as a function of the fundamental frequency $\left(f_{0}\right)$ and the grounded electrode shift velocity $(v)$. Light and dark gray solid lines show the apparent modification of the dispersion branches frequency boundaries at the $X$ and $\Gamma$ points, respectively. 
the first gap at $X$. Frequency shifts of the higher Bragg bandgaps at $X$ are also visible. Small gaps open up for large values of $v$ slightly below frequencies corresponding to shifted eigenfrequencies at $\Gamma$. Additionally, if the lines corresponding to the approximate frequency shift of the first gap at $X$ are drawn in Fig. 3(b) (solid gray lines), it can be verified that they give a good approximation of the dark area visible in the FT results at point B (Fig. 3(c)).

We have investigated the propagation of longitudinal acoustic waves through a 1D PnC made of identical piezoelectric elements separated by thin metallic electrodes connected to the ground. Attention was focused on cases where the locations of the electrical connections change as a function of time. We have shown that this spatio-temporal modulation of the electrical boundary conditions results in significant nonlinear effects analogous to the classical acousto-optical Brillouin scattering. Due to this scattering effect, a single input frequency produces multiple harmonics in the output. Since the amplitude of these harmonics reaches rather high values, this effect could be exploited to realize up (or down) frequency conversions of an input signal, which can be controlled by the modulation frequency. Non-reciprocal wave propagation was also achieved inside the piezoelectric modulated structure, with transmission contrasts reaching 40-50 dB in some cases. One important advantage of this type of system is that it can be easily controlled using transistor-based switching circuits. This enables real-time tuning of characteristics such as the gap position, the non-reciprocity parameters, or the inter-frequency energy transfer (from $f_{0}$ to the various $f_{n}^{ \pm}$). In order to get a clearer picture of the system's behavior, we have chosen a case with 40 electrodes in each PnC period. However, additional simulations have shown that similar effects can be evidenced with only a few electrodes per period. Beyond the effects highlighted here, this structure may serve as a unique platform to investigate a large variety of unusual and intriguing wave propagation phenomena.

See supplementary material for the procedure used to construct an approximation of the band diagram for the timedependent crystal.
This work has been performed in the frame of the ANR (French Research Agency) Project MIRAGES ANR-12BS09-0015. P.A.D. acknowledges the financial support from NSF Award No. 1640860.

${ }^{1}$ J. W. Strutt (Lord Rayleigh), Philos. Mag. Ser. 5(24), 145 (1887).

${ }^{2}$ J. D. Joannopoulos, S. G. Johnson, J. N. Winn, and R. D. Meade, Photonic Crystals: Molding the Flow of Light, 2nd ed. (Princeton University Press, Princeton/Oxford, 2008).

${ }^{3}$ Acoustic Metamaterials and Phononic Crystals, Springer Series in SolidState Sciences, edited by P. A. Deymier (Springer, Berlin, 2013), Vol. 173.

${ }^{4}$ J. R. Zurita-Sánchez, P. Halevi, and J. C. Cervantes-González, Phys. Rev. A 79, 053821 (2009).

${ }^{5}$ D. W. Wright and R. S. C. Cobbold, Smart Mater. Struct. 18, 015008 (2009).

${ }^{6}$ X. Dong, Y. Ye, B. Wang, C. Qiu, M. Ke, and Z. Liu, "Experimental demonstration of the acoustic frequency conversions by temporal phononic crystals," e-print arXiv:1305.1550v1[cond-mat.mtrl-sci].

${ }^{7}$ L. Brillouin, Ann. Phys. (Paris) 17, 88 (1922).

${ }^{8}$ F. Li, C. Chong, J. Yang, P. G. Kevrekidis, and C. Daraio, Phys. Rev. E 90, 053201 (2014).

${ }^{9}$ E. J. Reed, M. Soljačić, and J. D. Joannopoulos, Phys. Rev. Lett. 90, 203904 (2003).

${ }^{10}$ F. Cai, Z. He, A. Zhang, Y. Ding, and Z. Liu, Phys. Lett. A 374, 3971 (2010).

${ }^{11}$ E. S. Cassedy and A. A. Oliner, Proc. IEEE 51, 1342 (1963).

${ }^{12} \mathrm{Z}$. Yu and S. Fan, Nat. Photonics 3, 91 (2009).

${ }^{13}$ D.-W. Wang, H.-T. Zhou, M.-J. Guo, J.-X. Zhang, J. Evers, and S.-Y. Zhu, Phys. Rev. Lett. 110, 093901 (2013).

${ }^{14}$ N. Swinteck, S. Matsuo, K. Runge, J. O. Vasseur, P. Lucas, and P. A. Deymier, J. Appl. Phys. 118, 063103 (2015).

${ }^{15}$ M. B. Zanjani, A. R. Davoyan, A. M. Mahmoud, N. Engheta, and J. R. Lukes, Appl. Phys. Lett. 104, 081905 (2014).

${ }^{16}$ G. Trainiti and M. Ruzzene, New J. Phys. 18, 083047 (2016).

${ }^{17}$ S. Degraeve, C. Granger, B. Dubus, J. O. Vasseur, M. Pham Thi, and A.C. Hladky-Hennion, J. Appl. Phys. 115, 194508 (2014).

${ }^{18}$ A. Taflove, Computational Electrodynamics-The Finite Difference Time Domain method (Artech House Inc., Norwood, USA, 1995).

${ }^{19}$ The physical characteristics of PZT are the mass density $\rho_{0}=7450 \mathrm{~kg}$ $\mathrm{m}-3$, the elastic stiffness modulus at constant electric field $c^{E}=$ 4.3486.10 $10 \mathrm{~N} \mathrm{~m}-2$, the piezoelectric constant $\mathrm{e}=25 \mathrm{C} \mathrm{m}-2$, and the dielectric permittivity constant at constant strain $\varepsilon^{S}=9.4034 .10^{-9} \mathrm{~F}$ $\mathrm{m}-1$. The speed of longitudinal waves in bulk PZT is then $c_{0}=$ $\sqrt{\left(c^{E}+e^{2} / \varepsilon^{S}\right) / \rho_{0}}=3842 \mathrm{~m} / \mathrm{s}$.

${ }^{20}$ Y. Li, O. Bou Matar, V. Preobrazhensky, and P. Pernod, in 2008 IEEE International Ultrasonics Symposium Proceedings (2008), p. 1568. 